\title{
用于在超临界流体 $\mathrm{CO}_{2}$ 中直接溶解镧系和钣系 元素氧化物的 TRPO-HNO 3 络合物的性质
}

\author{
段五华 * 朱礼洋 景 山朱永唂
}

(清华大学核能与新能源技术研究院, 北京 102201)

\begin{abstract}
摘要 研究了 $\mathrm{TRPO}-\mathrm{HNO}_{3}$ 络合物的一些性质. 实验结果表明, 当 $\mathrm{HNO}_{3} / \mathrm{TRPO}$ 初始体积比从 $1 / 7$ 增大到 $5 / 1$ 时, TRPO- $\mathrm{HNO}_{3}$ 络合物中 $\mathrm{HNO}_{3}$ 浓度从 2.12 增大到 $6.16 \mathrm{~mol} / \mathrm{L},\left[\mathrm{HNO}_{3}\right] /[\mathrm{TRPO}$ 比 从 0.93 增大到 3.38, 络合物中水含量的变化范围为 $0.97 \%$ 2.70\%. TRPO- $\mathrm{HNO}_{3}$ 络合物的比重、粘 度和表面张力随络合物中 $\mathrm{HNO}_{3}$ 浓度的变化而变化. 采用気锁定技术研究了 TRPO- $\mathrm{HNO}_{3}$ 络合物 的 NMR 光谱, 结果表明, 在 NMR 谱图中, 络合物中 $\mathrm{HNO}_{3}$ 和 $\mathrm{H}_{2} \mathrm{O}$ 的质子会快速交换, 出现一个 单峰, 而当 $\mathrm{TRPO}-\mathrm{HNO}_{3}$ 络合物溶入低介电常数的溶剂时, 通过 NMR 谱图可以发现有小的 $\mathrm{HNO}_{3}$ 液滴形成.
\end{abstract}

\section{关键词 TRPO- $\mathrm{HNO}_{3}$ 络合物 性质 超临界流体 $\mathrm{CO}_{2}$ 溶解}

铀是核能的主要燃料, 其中 U-235 是产生能量的 裂变源. 在产生核能的过程中会产生裂变产物和超 铀元素 $\mathrm{Np}, \mathrm{Pu}, \mathrm{Am}$ 和 $\mathrm{Cm}$ 等. PUREX 流程是传统的 用于提取 $\mathrm{Pu}$ 和 $\mathrm{U}$ 的乏燃料后处理方法. 该流程首先 采用硝酸溶解乏燃料, 然后以 TBP-煤油为萃取剂从 酸性溶液中萃取 $\mathrm{U}$ 和 $\mathrm{Pu}$. 同时该流程也产生了大量 含有裂变产物和钣系元素的高放废液, 且处理这些 废液的成本很高.

中国提出了以 $\mathrm{TRPO}$ (三烷基氧膦)为萃取剂提取 高放废液中钶系和镧系裂变产物的 TRPO流程 $\frac{[1-4]}{\text {. }}$. TRPO萃取剂是工业产品, 价格低, 并且具有良好物 理特性、良好分离性能、良好辐照稳定性以及对钶系 和镧系元素有高的负载容量 ${ }^{[5]}$.

最近研究发现, TBP- $\mathrm{HNO}_{3}$ 络合物在超临界流体 $\mathrm{CO}_{2}\left(\mathrm{SCF}-\mathrm{CO}_{2}\right)$ 中能有效地溶解 $\mathrm{UO}_{2}$ 和 $\mathrm{U}_{3} \mathrm{O}_{8}$ 以及镧系 元素氧化物 ${ }^{[6-9]}$. 在此过程中, 固体 $\mathrm{UO}_{2}$ 中的 $\mathrm{U}(\mathrm{IV})$ 可能首先被 $\mathrm{TBP}-\mathrm{HNO}_{3}$ 络合物中 $\mathrm{HNO}_{3}$ 氧化成U(VI),
然后以 $\mathrm{UO}_{2}\left(\mathrm{NO}_{3}\right)_{2} \cdot 2 \mathrm{TBP}$ 形式溶入 $\mathrm{SCF}-\mathrm{CO}_{2}$, 最后通 过减压到大气压回收 $\mathrm{UO}_{2}\left(\mathrm{NO}_{3}\right)_{2} \cdot \mathrm{TBP}$. 整个过程不需 要任何水溶液来溶解氧化铀, 产生的废物少, 因此, 对于乏燃料后处理和铀污染的固体废物, 该方法很 有吸引力 ${ }^{[10,11]}$.

基于以上事实, 可以认为 $\mathrm{TRPO}-\mathrm{HNO}_{3}$ 络合物也 可用于在 $\mathrm{SCF}-\mathrm{CO}_{2}$ 中直接溶解镧系和钣系元素氧化 物. 为了掌握 TRPO-HNO 3 络合物在 $\mathrm{SCF}-\mathrm{CO}_{2}$ 中溶解 镧系和钶系元素氧化物的特性和机理, 本文首先研 究了用不同体积比的 $\mathrm{HNO}_{3}$ 和 TRPO 配制成的 TRPO- $\mathrm{HNO}_{3}$ 络合物的性质.

\section{1 实验方法}

\section{1 试剂}

TRPO(商业名Cyanex 923)购自美国Cyanamid公 司, 是四种三烷基氧膦的混合物, 其组成为 $\mathrm{R}_{3} \mathrm{PO}$ (14\%), $\mathrm{R}_{2} \mathrm{R}^{\prime} \mathrm{PO}(42 \%), \mathrm{RR}_{2}^{\prime} \mathrm{PO}(31 \%)$ 和 $\mathrm{R}_{3}^{\prime} \mathrm{PO}(8 \%), \mathrm{R}$ 
和 $\mathrm{R}^{\prime}$ 分别为正辛基和正己基 ${ }^{[5]}$. 为了减少原始 $\mathrm{TRPO}$ 中的水含量(约 4\%), 采用真空蒸馏法纯化, 使其水含 量减为 $0.2 \%$. TRPO的主要物理性质如表 1 所示.

表 1 TRPO 的性质

\begin{tabular}{cc}
\hline 性质 & 数值 \\
\hline 平均摩尔质量 & 348 \\
颜色 & 无色 \\
比重 $\left(25^{\circ} \mathrm{C}\right) / \mathrm{g} \cdot \mathrm{cm}^{-3}$ & 0.879 \\
粘度 $\left(25^{\circ} \mathrm{C}\right) / \mathrm{Pa} \cdot \mathrm{s}$ & 0.0328 \\
表面张力 $\left(25^{\circ} \mathrm{C}\right) / \mathrm{N} \cdot \mathrm{m}^{-1}$ & $27.5 \times 10^{-3}$ \\
水含量 $/ \%$ & 0.2 \\
闪点 $/{ }^{\circ} \mathrm{C}$ & 182 \\
凝固点 $/{ }^{\circ} \mathrm{C}$ & -5 \\
沸点 $/{ }^{\circ} \mathrm{C}$ & $180 \sim 225$ \\
水在 $\mathrm{TRPO}$ 的溶解度 $/ \mathrm{mg} \cdot \mathrm{L}^{-1}$ & 8 \\
$\mathrm{TRPO}$ 在水中的溶解度 $/ \mathrm{mg} \cdot \mathrm{L}^{-1}$ & 10 \\
\hline
\end{tabular}

$\mathrm{HNO}_{3}$ (分析纯, $15.5 \mathrm{~mol} / \mathrm{L}$ ), 北京化学试剂厂; $\mathrm{NaOH}$ (分析纯), 天津优尼莱博试剂公司.

\section{2 实验方法}

TRPO- $\mathrm{HNO}_{3}$ 络合物的制备：按一定体积比量取 TRPO 和 $15.5 \mathrm{~mol} / \mathrm{L}$ 的 $\mathrm{HNO}_{3}$ 放入一带塞子的玻璃试 管里剧烈混合 $30 \mathrm{~min}$, 然后离心分相 $1 \mathrm{~h}$, 最后分别 取出 TRPO 相和水相用于各性质测定. $\mathrm{HNO}_{3} / \mathrm{TRPO}$ 初始体积比为 $1: 7 \sim 5: 1$, 当 $\mathrm{HNO}_{3} / \mathrm{TRPO}$ 初始体积比 低于 $1: 7$ 时，所有的水相都溶入了有机相，因此没有 进行 $\mathrm{HNO}_{3} / \mathrm{TRPO}$ 初始体积比低于 $1: 7$ 的实验.

\section{3 分析方法}

水相中的 $\mathrm{HNO}_{3}$ 浓度用 $\mathrm{NaOH}$ 溶液滴定. TRPO$\mathrm{HNO}_{3}$ 络合物中 $\mathrm{HNO}_{3}$ 浓度也是用 $0.1 \mathrm{~mol} / \mathrm{L} \mathrm{NaOH}$ 溶 液滴定，在滴定前向络合物中加入过量的去离子水.

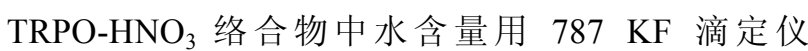
(Metrohm 公司, 瑞士)测定. TRPO- $\mathrm{HNO}_{3}$ 络合物的比 重通过称重法测定 (Mettler AE 200 天平, 瑞士).

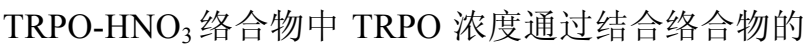
比重和 $\mathrm{H}_{2} \mathrm{O}$ 与 $\mathrm{HNO}_{3}$ 的浓度计算得到. TRPO- $\mathrm{HNO}_{3}$ 络合物的粘度和表面张力分别采用 Visco Basic Plus 粘度仪 (Fungilab, 西班牙)和 Model BZY 表面张力仪 (上海平衡仪器公司)测得. 用 $300 \mathrm{MHz}$ NMR 谱仪 (JOEL JNM-ECA300)测量 TRPO- $\mathrm{HNO}_{3}$ 络合物中的质 子 NMR 谱.

\section{2 结果与讨论}

\subsection{TRPO-HNO 3 络合物中 $\mathrm{HNO}_{3}$ 浓度和 $\mathrm{HNO}_{3} /$ TRPO 摩尔比}

两相中酸度随 $\mathrm{HNO}_{3} / \mathrm{TRPO}$ 初始体积比的变化如 图 1 所示. 当 $\mathrm{HNO}_{3} / \mathrm{TRPO}$ 初始体积比从 1:7 增大到

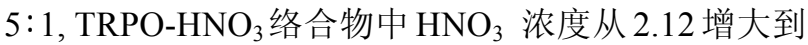
$6.16 \mathrm{~mol} / \mathrm{L}$, 平衡水相中酸度则从 1.55 增大到 15.18 $\mathrm{mol} / \mathrm{L} . \mathrm{TRPO}-\mathrm{HNO}_{3}$ 络合物中高的 $\mathrm{HNO}_{3}$ 浓度对于在 $\mathrm{SCF}-\mathrm{CO}_{2}$ 中溶解镧系和锕系元素氧化物是有利的.

从图 2 可得出, 当 TRPO- $\mathrm{HNO}_{3}$ 络合物中 $\mathrm{HNO}_{3}$ 浓度从 2.12 增大到 $6.16 \mathrm{~mol} / \mathrm{L}$, 络合物中 $\mathrm{HNO}_{3} / \mathrm{TRPO}$ 的摩尔比从 0.93 增大到 3.38 .

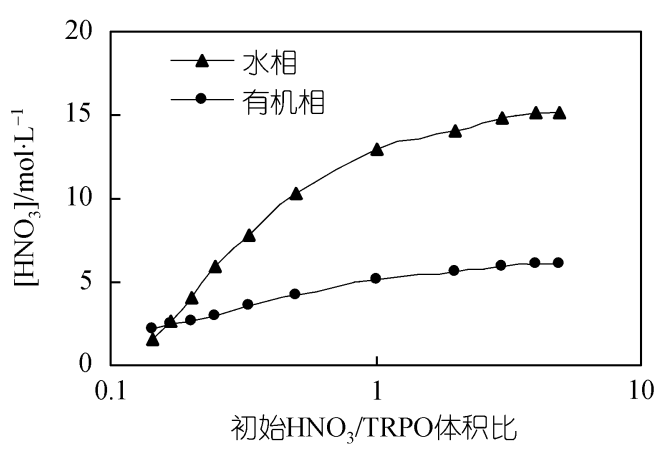

图 1 有机相和水相中 $\mathrm{HNO}_{3}$ 浓度

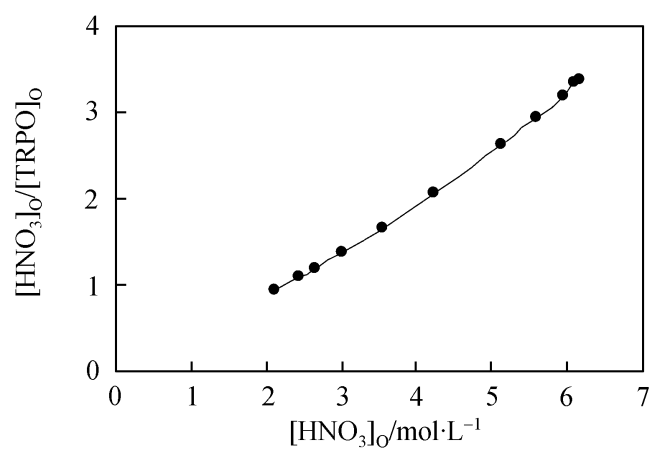

图 $2 \mathrm{TRPO}-\mathrm{HNO}_{3}$ 络合物中 $\mathrm{HNO}_{3} / \mathrm{TRPO}$ 的摩尔比 下标'O'表示 TRPO- $\mathrm{HNO}_{3}$ 络合物相

无机酸如 $\mathrm{HNO}_{3}$ 常常是不溶入 $\mathrm{CO}_{2}$ 的, 而通过与 能溶入 $\mathrm{CO}_{2}$ 的Lewis碱如TRPO络合后, 就可溶入 $\mathrm{CO}_{2}$ 了. 这种Lewis酸碱络合方法提供了一种可使各种不 溶入 $\mathrm{CO}_{2}$ 的酸能与 $\mathrm{SCF}-\mathrm{CO}_{2}$ 相中金属氧化物反应的 方法 ${ }^{[6]}$. 


\subsection{TRPO- $\mathrm{HNO}_{3}$ 络合物中 $\mathrm{H}_{2} \mathrm{O}$ 含量}

从图 3 可见, 随着 $\mathrm{TRPO}-\mathrm{HNO}_{3}$ 络合物中 $\mathrm{HNO}_{3}$ 浓度从 2.12 增大到 $2.65 \mathrm{~mol} / \mathrm{L}$, 络合物中 $\mathrm{H}_{2} \mathrm{O}$ 含量 从 $1.39 \%$ 降低到 $0.97 \%$, 而当络合物中 $\mathrm{HNO}_{3}$ 浓度从 2.65 增大到 $6.16 \mathrm{~mol} / \mathrm{L}$ 时, 络合物中 $\mathrm{H}_{2} \mathrm{O}$ 含量从 $0.97 \%$ 提高到 $2.70 \%$. 主要是因为络合物中 $\mathrm{HNO}_{3}$ 浓 度低时, $\mathrm{HNO}_{3}$ 会排挤络合物中的 $\mathrm{H}_{2} \mathrm{O}$, 而络合物中 $\mathrm{HNO}_{3}$ 浓度较高时, $\mathrm{H}_{2} \mathrm{O}$ 会与硝酸一起与 TRPO 进一 步络合, 从而使络合物中水含量增大.

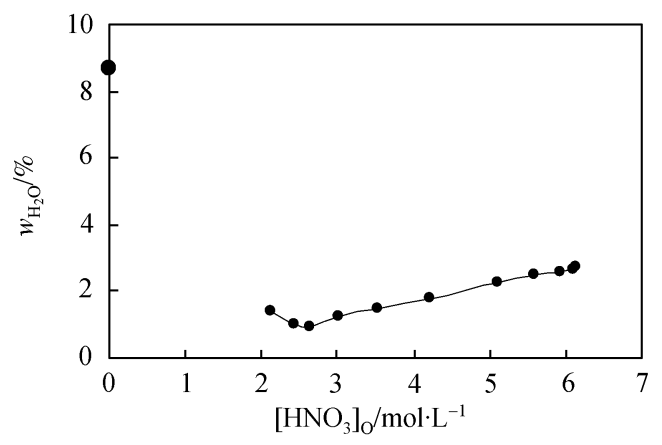

图 3 TRPO- $\mathrm{HNO}_{3}$ 络合物中的 $\mathrm{H}_{2} \mathrm{O}$ 含量 $\left(25^{\circ} \mathrm{C}\right)$

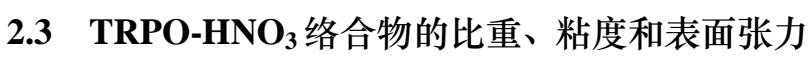

$\mathrm{TRPO}-\mathrm{HNO}_{3}$ 络合物的比重、粘度和表面张力如 表 2 所示. 从表 2 中可看出, 随着 $\mathrm{TRPO}-\mathrm{HNO}_{3}$ 络合 物中 $\mathrm{HNO}_{3}$ 浓度从 2.12 增大到 $6.16 \mathrm{~mol} / \mathrm{L}$, 络合物的 比重从 0.941 增大到 $1.051 \mathrm{~g} / \mathrm{cm}^{3}$, 粘度从 42.3 降低到 $12.71 \mathrm{mPa} \cdot \mathrm{s}$, 表面张力从 29.5 增大到 $32.3 \mathrm{mN} / \mathrm{m}$.

表 2 TRPO- $\mathrm{HNO}_{3}$ 络合物的比重、粘度和表面张力 $\left(25^{\circ} \mathrm{C}\right)$

\begin{tabular}{cccc}
\hline$\left[\mathrm{HNO}_{3}\right]_{\mathrm{O}} / \mathrm{mol} \cdot \mathrm{L}^{-1}$ & 比重 $/ \mathrm{g} \cdot \mathrm{cm}^{-3}$ & 粘度 $/ \mathrm{mPa} \cdot \mathrm{s}$ & 表面张力 $/ \mathrm{mN} \cdot \mathrm{m}^{-1}$ \\
\hline 6.16 & 1.051 & 12.71 & 32.3 \\
6.12 & 1.051 & 13.06 & 32.3 \\
5.95 & 1.050 & 13.54 & 32.3 \\
5.59 & 1.042 & 15.78 & 32.3 \\
5.12 & 1.027 & 18.18 & 32.1 \\
4.23 & 0.999 & 24.46 & 31.5 \\
3.55 & 0.982 & 29.63 & 31.2 \\
3.02 & 0.968 & 32.01 & 30.8 \\
2.65 & 0.956 & 34.30 & 30.4 \\
2.45 & 0.949 & 36.39 & 29.8 \\
2.12 & 0.941 & 42.30 & 29.5 \\
\hline
\end{tabular}

\subsection{TRPO-HNO 3 络合物的 NMR}

采用氝锁定技术进行了室温下TRPO- $\mathrm{HNO}_{3}$ 络合 物的 NMR 研究 ${ }^{[12,13]} . \mathrm{D}_{2} \mathrm{O}$ 放置在一直径小于 $\mathrm{NMR}$ 样
品管的小管中，插入放置了 TRPO- $\mathrm{HNO}_{3}$ 络合物的 NMR测试管. 作NMR测试的目的是为了研究络合物 中 $\mathrm{HNO}_{3}$ 和 $\mathrm{H}_{2} \mathrm{O}$ 质子的化学位移, 从而研究体系的化 学特征。采用気锁定技术后, 水饱和 TRPO和 TRPO- $\mathrm{HNO}_{3}$ 络合物的NMR谱分别如图 4 和图 5 所示. 图 4 中 $\delta 4.22$ 的单峰为水饱和 TRPO中 $\mathrm{H}_{2} \mathrm{O}$ 的质子峰, 图 5 中 $\delta 12.22$ 的单峰为 $\mathrm{TRPO}-\mathrm{HNO}_{3}$ 络合物中 $\mathrm{HNO}_{3}$ 和 $\mathrm{H}_{2} \mathrm{O}$ 的质子峰. 而图 4 和图 5 中 $\delta 4.71$ 的单峰是重 水中微量 $\mathrm{H}_{2} \mathrm{O}$ 的峰. 对采用不同 $\mathrm{HNO}_{3} / \mathrm{TRPO}$ 初始体 积比配制的TRPO- $\mathrm{HNO}_{3}$ 络合物都进行了相同的研究, 在所有 $N M R$ 谱中, TRPO- $\mathrm{HNO}_{3}$ 络合物中 $\mathrm{HNO}_{3}$ 和 $\mathrm{H}_{2} \mathrm{O}$ 的质子峰都是单峰. $\mathrm{HNO}_{3}-\mathrm{H}_{2} \mathrm{O}$ 质子单峰的化学位移 随TRPO- $\mathrm{HNO}_{3}$ 络合物中 $\mathrm{HNO}_{3}$ 浓度的变化如图 6 所 示. $\mathrm{HNO}_{3}-\mathrm{H}_{2} \mathrm{O}$ 质子单峰的化学位移首先随着 TRPO- $\mathrm{HNO}_{3}$ 络合物中 $\mathrm{HNO}_{3}$ 浓度的增大而增大, 然 后又随着络合物中 $\mathrm{HNO}_{3}$ 浓度的增大而稍微减小, 最 后当络合物中 $\mathrm{HNO}_{3}$ 浓度大于 $5.95 \mathrm{~mol} / \mathrm{L}$ 时基本保持 不变.

由于 $\mathrm{CHCl}_{3}$ 在常温下的介电常数小, 因此选择 $\mathrm{CDCl}_{3}$ 为溶剂来评价超临界流体 $\mathrm{CO}_{2}$ 对络合物的抗溶

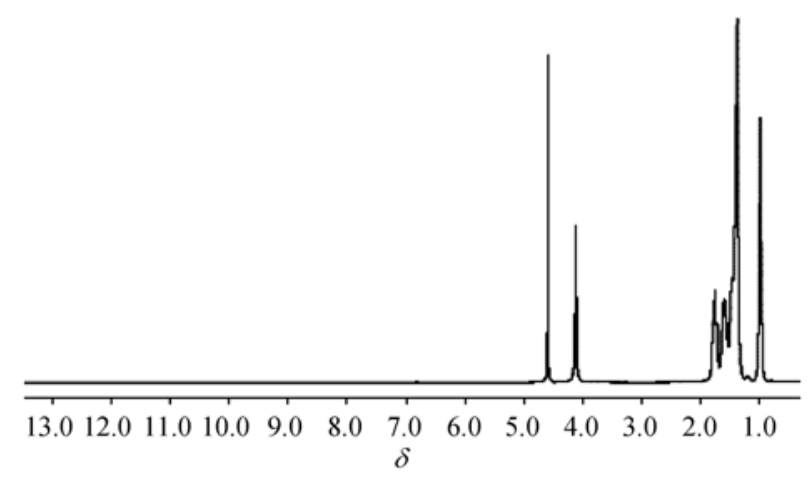

图 4 水饱和 TRPO 的 ${ }^{1} \mathrm{H}$ NMR 谱

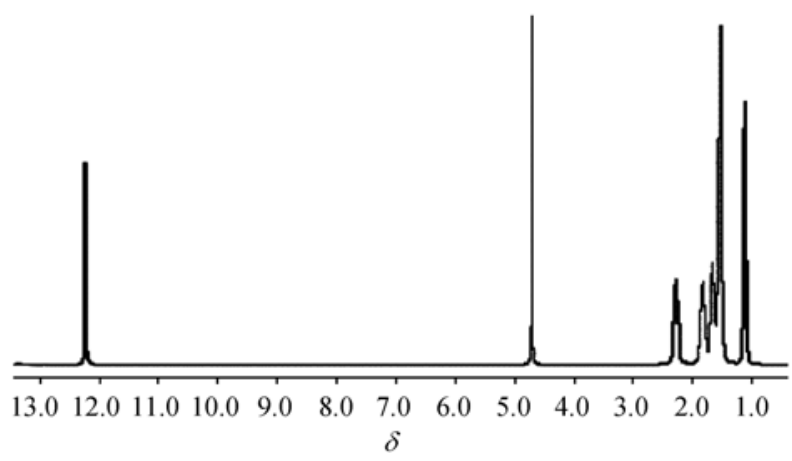

图 5 TRPO- $\mathrm{HNO}_{3}$ 络合物的 ${ }^{1} \mathrm{H}$ NMR 谱 $\mathrm{HNO}_{3} /$ TRPO 初始体积比为 $1 / 1$ 


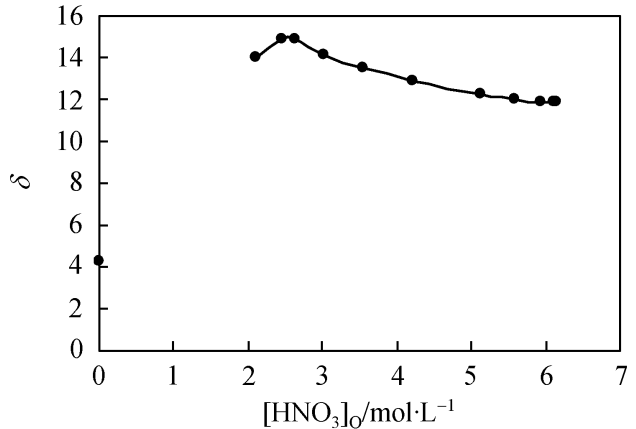

图 $6 \mathrm{H}_{2} \mathrm{O}$ 质子峰的化学位移随 TRPO- $\mathrm{HNO}_{3}$ 络合物中 $\mathrm{HNO}_{3}$ 浓度的变化

剂效应. 在 $\mathrm{CDCl}_{3}$ 中 $\mathrm{TRPO}-\mathrm{HNO}_{3}$ 络合物的 $\mathrm{NMR}$ 谱 如图 7 所示. 从图 7 中可见, 除了烷基基团的一些质 子峰外, 还有两个峰, $\delta 6.84$ 的峰为体系产生的硝酸 液滴峰, 而 $\delta 14.14$ 的峰是络合物在 $\mathrm{CDCl}_{3}$ 中 $\mathrm{HNO}_{3}$ $\mathrm{H}_{2} \mathrm{O}$ 的质子峰. 硝酸液滴不溶入 $\mathrm{CDCl}_{3}$ 溶液, 因而可 通过采用不同浓度硝酸与 $\mathrm{CDCl}_{3}$ 混合的单独 $\mathrm{NMR}$ 研 究来确认 $\delta 6.84$ 的峰, 而且在 $\mathrm{CDCl}_{3}$ 中的硝酸质子峰 随着酸性溶液中硝酸浓度的增大而向高场位移. $\mathrm{CDCl}_{3}-\mathrm{TRPO}-\mathrm{HNO}_{3}$ 络合物体系的 NMR 结果定性地 表明，当 TRPO- $\mathrm{HNO}_{3}$ 络合物溶入低介电常数的溶剂 时会产生硝酸小液滴，因而表明超临界流体 $\mathrm{CO}_{2}$ 对 TRPO- $\mathrm{HNO}_{3}$ 络合物有抗溶剂效应. 硝酸液滴开始可 能很小，而增大到一定尺寸时将使溶液混浊.

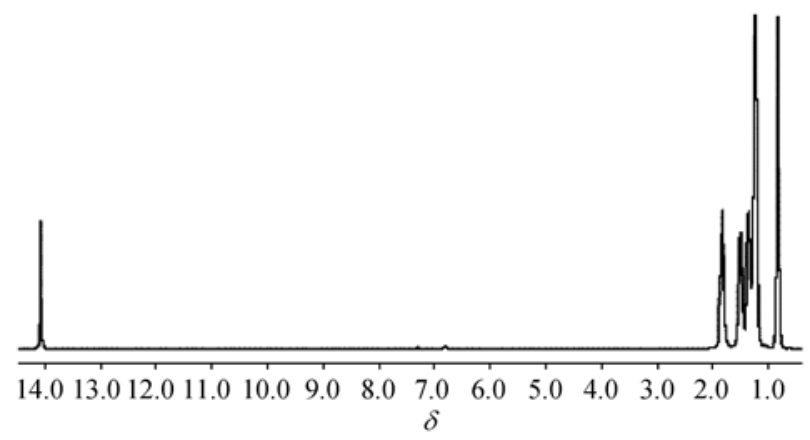

图 7 在 $\mathrm{CDCl}_{3}$ 中 $\mathrm{TRPO}-\mathrm{HNO}_{3}$ 络合物的 ${ }^{1} \mathrm{H} \mathrm{NMR}$ 谱 $\mathrm{HNO}_{3} / \mathrm{TRPO}$ 初始体积比为 $1 / 4$

\section{3 结论}

研究了 $\mathrm{TRPO}-\mathrm{HNO}_{3}$ 络合物的一些性质包括 $\mathrm{HNO}_{3}$ 浓度、 $\mathrm{H}_{2} \mathrm{O}$ 含量、比重、粘度、表面张力和 NMR. 络合物含有高浓度 $\mathrm{HNO}_{3}$ 有利于在超临界流体中溶 解镧系和钣系元素氧化物. 络合物中的 $\mathrm{H}_{2} \mathrm{O}$ 也有助
于这些氧化物的溶解. 在 NMR 谱图中, 络合物中 $\mathrm{HNO}_{3}$ 和 $\mathrm{H}_{2} \mathrm{O}$ 的质子会快速交换，出现一个单峰，而 且, 当 TRPO- $\mathrm{HNO}_{3}$ 络合物溶入低介电常数的溶剂时, 通过 NMR 谱图可以发现有小的 $\mathrm{HNO}_{3}$ 液滴形成，这 个现象在今后研究中应加以重视.

\section{参考文献}

1 Zhu Y J, Song C L. Recovery of Np, Pu, and Am from highly active waste: trialkylphosphine oxide extraction. In: Morss L R, Fuger J. eds. Transuranium Elements: A Half Century. American Chemical Society, Washington, DC, 1992. 318-330

2 Zhu Y J, Jiao R Z. Chinese experience in the removal of actinides from highly active waste by trialkylphosphine-oxide extractant. Nucl Technol, 1994, 108: 361-369

3 Wang J C, Song C L. Hot test of trialkyl phosphine oxide (TRPO) for removing actinides from highly saline high-level liquid waste (HLLW). Solvent Extr Ion Exc, 2001, 19(2): 231-242[DOI]

4 Song C L, Jin G Y, Wang J C, Zhu Y J. Treatment of Chinese HLLW by partitioning. Tsinghua Sci Technol, 1996, 1(1): 81-85

5 Euzebiusz D, Jan S. Composition of CYANEX 923, CYANEX 925, CYANEX 921 and TOPO. Solvent Extr Ion Exc, 1998, 16(6): 1515-1525[DOI]

6 Tomioka O, Meguro Y, Enokida Y, Yamamoto I, Yoshida Z. Dissolution behavior of uranium oxides with supercritical $\mathrm{CO}_{2}$ using $\mathrm{HNO}_{3}$-TBP complex as a reactant. J Nucl Sci Technol, 2001, 38(12): 1097-1102

7 Tomioka O, Enokida Y, Yamamoto I. Selective recovery of neodymium from oxides by direct extraction method with supercritical $\mathrm{CO}_{2}$ containing TBP- $\mathrm{HNO}_{3}$ complex. Sep Sci Technol, 2002, 37(5): 1153-1162[DOI]

8 Enokida Y, El-Fatah S A, Wai C M. Ultrasound-enhanced dissolution of $\mathrm{UO}_{2}$ in supercritical $\mathrm{CO}_{2}$ containing a $\mathrm{CO}_{2}$-philic complexant of tri- $n$-butylphosphate and nitric acid. Ind Eng Chem Res, 2002, 41(9): 2282-2286[DOI]

9 Samsonov M D, Trofimov T I, Vinokurov S E, Lee S C, Myasoedov B F, Wai C M. Dissolution of actinide oxides in supercritical fluid carbon dioxide containing various organic ligands. J Nucl Sci Technol, 2002, 3(Suppl): 263-266

10 Meguro Y, Iso S, Yoshida Z, Tomioka O, Enokida Y, Yamamoto I. Decontamination of uranium oxides from solid wastes by supercritical $\mathrm{CO}_{2}$ fluid leaching method using $\mathrm{HNO}_{3}$-TBP complex as a reactant. J Supercrit Fluid, 2004, 31: 141-147[DOI]

11 Shimada T, Ogumo S, Ishihara N, Kosaka Y, Mori Y. A study on the technique of spent fuel reprocessing with supercritical fluid direct extraction method (Super-DIREX method). J Nucl Sci Technol, 2002, Supplement: 757-760

12 Enokida Y, Tomioka O, Lee S C, Rustenholtz A, Wai C M. Characterization of a tri- $n$-butyl phosphate-nitric acid complex: a $\mathrm{CO}_{2}$-soluble extraction for dissolution of uranium dioxide. Ind Eng Chem Res, 2003, 42(21): 5037-5041[DOI]

13 Samsonov M D, Wai C M, Lee S C, Kulyako Y, Smart N G. Dissolution of uranium dioxide in supercritical fluid carbon dioxide. Chem Commun, 2001, 1868-1869 